\title{
Concentraciones de albúmina modificada por la isquemia en niños con asma: estudio piloto
} Ischemia-modified albumin levels in children with asthma: a pilot study

\author{
Dr. Mahmut Dogrua, Dra. Handan Akoglu ${ }^{a}$ Dr. Muhammet F. Kilinckayab $y$ \\ Dra. Gozde Ulferc
}

\section{RESUMEN}

Introducción. La albúmina modificada por la isquemia puede aumentar en el asma (IMA), estrés oxidativo y la inflamación. El objetivo fue evaluar las concentraciones de IMA en niños asmáticos durante períodos asintomáticos y de exacerbación.

Población y métodos. Niños asmáticos y sanosen seguimiento (grupo de referencia). La gravedad dela exacerbación seevaluómediantela Iniciativa global para el asma (GINA) y la puntuación del índice pulmonar modificado (MPIS). Se usaron pruebas intraepidérmicas y de proteína C reactiva para medir las concentraciones séricas de IMA durante la exacerbación y 4 semanas después del tratamiento.

a. Universidad de Ciencias de la Salud, Hospital de Formación e Investigación de Enfermedades Pediátricas y de la Mujer Zeynep Kamil, Departamento de Pediatría, Estambul, Turquía.

b. Hospital de Formación e Investigación Ankara Numune, Departamento de Bioquímica, Ankara, Turquía.

c. Istanbul Medipol University, Departamento de Bioquímica, Estambul, Turquía.

Correspondencia:

Prof. Asoc. Dr. Mahmut Dogru:

mdmahmut@yahoo. com

Financiamiento: Ninguno.

Conflicto de intereses: Ninguno que declarar.

Recibido: 7-8-2017

Aceptado: 29-1-2018
Cómo citar: Dogru M, Akoglu H, Kilinckaya M, Ulfer G. Concentraciones de albúmina modificada por la isquemia en niños con asma: estudio piloto. Arch Argent Pediatr 2018;116(4):e522-e528. http: / / dx.doi.org/10.5546/ aap.2018.e522

Texto completo en inglés:

http: / / dx.doi.org/10.5546/ aap.2018.eng.e522

\section{INTRODUCCIÓN}

Se define asma como la repetición de síntomas respiratorios como sibilancias, falta de aire, opresión en el pecho y tos, que varían con el tiempo y en intensidad, junto con una limitación variable del flujo de aire en la exhalación. La exacerbación del asma se caracteriza por la disminución progresiva de la función pulmonar y el aumento paulatino de los síntomas. La característica principal de una exacerbación del asma es la obstrucción del flujo de aire en las vías respiratorias. Para determinar la gravedad de la exacerbación del asma, la hipoxia que provoca la obstrucción de las vías respiratorias se evalúa midiendo la saturación de oxígeno. El objetivo del tratamiento es contrarrestar rápidamente la obstrucción del flujo de aire y la hipoxemia, abordar la patofisiología inflamatoria subyacente y evitar las recaídas. La primera medida para tratar la exacerbación del asma en el departamento de emergencias, es administrar oxígeno mediante una mascarilla para lograr y mantener la saturación percutánea de oxígeno entre el $94 \%$ y el $98 \%{ }^{1}$

El extremo terminal del aminoácido en la albúmina tiene la capacidad de unirse a metales pesados (cobre, níquel y cobalto). En condiciones patológicas como la hipoxia o la acidosis, el daño al radical libre que tiene lugar en caso de isquemia reduce la capacidad de unión de los metales al dominio N-terminal de la albúmina. Fueron Bar-Or et $a^{2} .^{2}$ quienes detectaron por primera vez esa reducción en la capacidad 
de unión del cobalto con el dominio N-terminal de la albúmina en pacientes con infarto de miocardio, y desarrollaron la prueba de la unión albúmina-cobalto conocida como "albúmina modificada por la isquemia (IMA)". La IMA se ha estudiado exhaustivamente en cardiopatías isquémicas, y la Administración de Alimentos y Medicamentos (FDA) aprobó su medición para evaluar la magnitud de la isquemia cardíaca. La albúmina modificada por la isquemia está aceptada como indicador del estrés oxidativo. ${ }^{2-4}$ Además de las enfermedades relacionadas con la isquemia, se encontró que en los pacientes con fiebre reumática, ${ }^{5}$ enfermedad pulmonar obstructiva crónica (EPOC), ${ }^{6}$ enfermedad intestinal inflamatoria ${ }^{7}$ y síndrome de Behçet, ${ }^{8}$ las concentraciones de IMA eran más altas que en los controles. El asma puede provocar hipoxia durante una exacerbación, pero la información con que contamos sobre las concentraciones de IMA en casos de asma es limitada. En Turquía, Karagol et al., ${ }^{9}$ evaluaron las concentraciones de IMA durante las exacerbaciones de asma y encontraron que esas concentraciones eran más elevadas en los pacientes que en los controles. Encontraron, además, que no había diferencias significativas entre las concentraciones de IMA en los controles y en los pacientes asmáticos que se evaluaban 2 semanas después de la exacerbación.

El objetivo de este estudio fue comparar las concentraciones de IMA en niños asmáticos durante el período asintomático y durante las exacerbaciones de asma con las concentraciones de IMA en niños sanos.

\section{POBLACIÓN Y MÉTODOS}

Población del estudio: participaron en el estudio los niños asmáticos que ingresaron consecutivamente al departamento de emergencias debido a una exacerbación del asma entre diciembre de 2015 y marzo de 2016. Se excluyeron del estudio los pacientes con obesidad (índice de masa corporal > percentil 95) $)^{10}$ y los pacientes con enfermedades crónicas, como fibrosis quística o bronquiectasia. Los niños sin ninguna enfermedad alérgica o crónica que habían sido derivados al consultorio pediátrico general para el control de rutina de pacientes ambulatorios y a quienes se les realizaban análisis de sangre para otras pruebas (hemograma completo, urea, creatinina, etc.) se incorporaron como grupo de referencia.

Diseño del estudio: en este estudio transversal prospectivo, se pidió a los pacientes que rellenaran un cuestionario durante la primera evaluación. En este cuestionario se incluyen datos demográficos de los pacientes e información sobre el uso de antiinflamatorios, la presencia de infección de las vías respiratorias altas (IVRA), los síntomas previos a la exacerbación, la cantidad de exacerbaciones y hospitalizaciones en el último año, y los antecedentes de atopia.

Primera evaluación: durante la exacerbación del asma, se evaluó a los pacientes clínicamente mediante la Iniciativa global para el asma (GINA) y la Puntuación del índice pulmonar modificado (MPIS), y con análisis de laboratorio (IMA y proteína $C$ reactiva $[P C R]$ ). Durante la exacerbación, se realizó una extracción sanguínea a cada paciente y se conservó una muestra de $5 \mathrm{ml}$ de suero a $-20{ }^{\circ} \mathrm{C}$. La proteína $\mathrm{C}$ reactiva (PCR) se midió en el plazo de 2 horas después de dicha extracción.

Segunda evaluación: las muestras de sangre de los pacientes para la determinación de la IMA se tomaron por lo menos 4 semanas después del tratamiento para exacerbaciones agudas y durante el período de ausencia de síntomas.

Aspectos éticos: el Hospital de Formación e Investigación de Enfermedades Pediátricas y de la Mujer Zeynep Kamil otorgó la aprobación ética para este estudio; el objetivo del estudio y los procedimientos se explicaron a los niños y sus familias, y se obtuvo su consentimiento verbal. El estudio se llevó a cabo de conformidad con los principios de la declaración de Helsinki.

\section{Evaluación clínica}

La gravedad de la exacerbación del asma en los pacientes se categorizó según la Iniciativa global para el asma (GINA) como leve/moderada y grave. ${ }^{1}$ Además de esta categorización, en el momento de la admisión se calculó la Puntuación del índice pulmonar modificado (MPIS), que se utilizó en la evaluación clínica de los pacientes. En la MPIS se evalúan 6 categorías: saturación de oxígeno, uso del músculo accesorio, relación entre el flujo inspiratorio y el flujo espiratorio, intensidad de las sibilancias, frecuencia cardíaca y frecuencia respiratoria. A cada una de estas 6 mediciones u observaciones se le asignó una puntuación de 0 a 3 (Tabla 1$).{ }^{11}$

\section{Análisis de la muestra de sangre}

La prueba de unión al cobalto se usó para la medición de la IMA según la definición de Bar-Or et al., ${ }^{2}$ El principio de esta prueba reside en la determinación colorimétrica del complejo de ditiotreitol (DTT) y cobalto no ligado. Se 
añadieron $50 \mu 1$ de cloruro de cobalto al $0,1 \%$ a $200 \mu \mathrm{l}$ del suero del paciente, se mezcló con agitadora vorticial y se incubó durante 10 minutos para posibilitar la unión de la albúmina con el cobalto. Se añadieron $50 \mu 1$ de solución de $1,5 \mathrm{mg} / \mathrm{ml}$ de DTT para permitir la reacción del cobalto no ligado durante 2 minutos. Después de este tiempo, se completó la reacción agregando $\mathrm{NaCl}$ al 0,9\%. Se siguió el mismo procedimiento para el blanco de la muestra, pero se usó agua destilada en lugar de DTT. Al terminar la reacción, se determinaron los niveles de absorbencia a $470 \mathrm{~nm}$ en un espectrofotómetro Shimadzu UV-1201 V. La diferencia entre la muestra y el blanco de la muestra se registró como valor de la IMA. Los coeficientes de variación (CV\%) intraensayo e interensayo de los métodos fueron, respectivamente, 3,20 y 3,91.

Las concentraciones de proteína $\mathrm{C}$ reactiva se midieron mediante inmunonefelometría, con el sistema BN ProSpec (Dade Behring Inc., Illinois, EE. UU.).

\section{Prueba intraepidérmica}

Las pruebas intraepidérmicas de los pacientes se llevaron a cabo cuando se presentaron por primera vez en nuestro departamento de alergias. Se utilizaron aeroalérgenos comunes (Dermatophagoides pteronyssinus, Dermatophagoides farinea, Alternaria alternaria), cucarachas (Blatella germanica), caspa de perro y de gato, mezcla de polen de las gramíneas (Lollium perenne, Dactylis glomerata, Phleum pratense, Anthoxanthum odaratum, Poa pratensis, Festuca eliator, Agrostis vulgaris, Holcus lanatus, Cynodon dactylon, Avena sativa, Avena fatua, Lotus Corniculatus), mezcla de cereales (avena, trigo, cebada, maíz), mezcla de polen de árboles (Acer pseudoplanatus, Aesculus hippocastanum, Robinia pseudoacacia, Tilia platyphyllos, Platanus vulgaris), mezcla de polen de hierbas
(Medicago sativa, Trifolium pratense, Brassica nigra, Urtica dioica, Rumex acetosa), (Stallergenes SA, 92160 Antony, Francia). Las pruebas se realizaron con una lanceta en la cara anterior del antebrazo. Se usaron histamina $(10 \mathrm{mg} / \mathrm{ml})$ y solución fisiológica como referencias positiva y negativa, respectivamente. Las reacciones cutáneas se evaluaron 20 minutos después de aplicar la prueba en la piel, y las zonas induradas $\geq 3 \mathrm{~mm}$ se consideraron indicadoras de reacción positiva.

\section{Análisis estadístico}

El tamaño de la muestra se calculó en $\mathrm{N}=72$. Para el análisis estadístico se utilizó el paquete estadístico para ciencias sociales (Statistical Package for Social Sciences, SPSS), versión 15.0. Los niveles para las variables continuas se presentaron como la media \pm desviación estándar o como la mediana (intervalo intercuartílico) en función de la normalidad de la distribución. Se usó la prueba $t$ de Student para comparar la distribución normal y homogénea de los niveles paramétricos. Los niveles no paramétricos se compararon usando la prueba $\chi^{2}$ y la prueba U de Mann-Whitney. Las pruebas de correlación de Pearson y Spearman se usaron en función de la normalidad de los niveles. Para comparar las variables dependientes (exacerbaciones y estadio estable) se usó la prueba del orden con signo de Wilcoxon. Para evaluar los resultados se aceptaron un intervalo de confianza del 95\% y un nivel de significación $p<0,05$.

\section{RESULTADOS}

En total, se incluyeron 52 niños en el estudio. El grupo del estudio estuvo formado por 26 niños con asma (promedio de edad: 6,05 $\pm 2,15$ años; 17 varones y 9 mujeres) y 26 niños como grupo de referencia (promedio de edad: $6 \pm 2,12$ años; 15 varones y 11 mujeres). No hubo diferencias

TABLA 1. Puntuación del índice pulmonar modificado ${ }^{11}$

\begin{tabular}{|c|c|c|c|c|c|c|c|c|}
\hline & Frecuencia & ardíaca/min & Frecuencia re & spiratoria/min & & & Sibilancia & \\
\hline & $<3$ años de edad & $\geq 3$ años de edad & $<6$ años de edad & $\geq 6$ años de edad & accesorio & exhalación & & $\%$ (ambiente) \\
\hline 0 & $<120$ & $<100$ & $\leq 30$ & $\leq 20$ & No & $2: 1$ & No & $\geq 96$ \\
\hline 1 & $120-140$ & $100-120$ & $31-45$ & $21-35$ & Leve & $1: 1$ & Final de la espiración & $93-95$ \\
\hline 2 & $141-160$ & $121-140$ & $46-60$ & $36-50$ & Moderado & $1: 2$ & $\begin{array}{c}\text { Sibilancia inspiratoria } \\
\text { y espiratoria } \\
\text { buena oxigenación }\end{array}$ & $90-92$ \\
\hline 3 & $>160$ & $>140$ & $>60$ & $>50$ & Grave & $1: 3$ & $\begin{array}{l}\text { Sibilancia inspiratoria } \\
\text { y espiratoria } \\
\text { oxigenación reducida }\end{array}$ & $<90$ \\
\hline
\end{tabular}


estadísticamente significativas respecto de la edad y el sexo entre los pacientes y el grupo de referencia ( $p=0,776$ y $p=0,938$, respectivamente). El nivel medio de IMA fue de 0,45 $\pm 0,12$ unidades de absorbencia (ABSU) $(0,206-0,721)$ en el grupo objeto de estudio y de 0,32 $\pm 0,08$ ABSU $(0,145$ $0,435)$ en el grupo de referencia. La diferencia fue estadísticamente significativa $(p=0,001)$.

Diecinueve niños recibían tratamiento antiinflamatorio (esteroides para inhalar y/o montelukast) en forma regular. La diferencia en las concentraciones de IMA entre los niños que recibían y los que no recibían tratamiento antiinflamatorio no fue estadísticamente significativa $(p=0,692)$, aunque la concentración media de IMA en los niños que sí recibieron tratamiento antiinflamatorio fue más baja que en los otros niños. Diecinueve pacientes presentaban síntomas de IRA antes de las exacerbaciones de asma. Al comparar las concentraciones de IMA en los niños que presentaban síntomas de IRA con las de los niños que no presentaban esos síntomas, encontramos que las concentraciones de IMA eran más elevadas, aunque no significativas estadísticamente, en los niños con síntomas de IRA $(p=0,651)$. La concentración de PCR en los pacientes con exacerbación del asma fue normal $(<0,5 \mathrm{mg} / \mathrm{dl})$. No hubo diferencias en las concentraciones de IMA entre los niños con altas concentraciones de PCR y los niños con concentraciones de PCR bajas $(p=0,742)$. En 21 niños, la prueba intraepidérmica resultó positiva. No se observaron diferencias significativas al comparar las concentraciones de IMA en los niños asmáticos con sensibilidad a las pruebas intraepidérmicas con las de los niños que no mostraron sensibilidad $(p=0,111)$. En términos de la clasificación GINA, 23 niños presentaron asma de intensidad leve a moderada. La comparación de las concentraciones de IMA en función de la gravedad de la exacerbación del asma mostraron valores de IMA notablemente más elevados en los pacientes con exacerbaciones graves $(p=0,009)$ (Tabla 2$)$.

La concentración media de IMA en los niños asmáticos durante el período asintomático, al menos 4 semanas después de las exacerbaciones fue 0,41 $\pm 0,14$ ABSU $(0,151$ - 0,699). En comparación con el grupo de referencia, la concentración media de IMA en el período de estabilidad fue notablemente más alta en el grupo de los pacientes $(p=0,005)$. En los pacientes asmáticos, las concentraciones de IMA en el período de estabilidad fue menor que durante las exacerbaciones; sin embargo, esta diferencia no fue estadísticamente significativa $(p=0,077)$.

La puntuación MPIS media de los pacientes fue $7 \pm 2,68$ (3-15). Se observó una correlación fronteriza positiva entre los valores del MPIS y las concentraciones de IMA $(r=0,376, p=0,05)$.

No se observó correlación entre las concentraciones de IMA y el ingreso a la sala de emergencias durante el último año, las hospitalizaciones o las concentraciones de PCR. Observamos una relación negativa pero no estadísticamente significativa entre la saturación

TABLA 2. Comparación de la relación entre los parámetros clinicos/de laboratorio y la albúmina modificada por la isquemia en exacerbaciones de asma

\begin{tabular}{|c|c|c|c|}
\hline Parámetros & $\mathbf{n}$ & Albúmina modificada por la isquemia* & $P$ \\
\hline \multicolumn{4}{|c|}{ Tratamiento administrado antes de las exacerbaciones, $\mathrm{n}(\%)$} \\
\hline Sí & $19(73,1)$ & $0,44(0,39-0,47)$ & \\
\hline No & $7(26,9)$ & $0,45(0,39-0,58)$ & $0,692^{* *}$ \\
\hline \multicolumn{4}{|c|}{ IRA síntomas antes de las exacerbaciones, $\mathrm{n}(\%)$} \\
\hline Sí & $19(73,1)$ & $0,45(0,39-0,55)$ & \\
\hline No & $7(26,9)$ & $0,44(0,37-0,47)$ & $0,651^{* *}$ \\
\hline \multicolumn{4}{|c|}{ Elevación de la PCR, n (\%) } \\
\hline Sí & $12(46,2)$ & $0,45(0,34-0,55)$ & \\
\hline No & $14(53,8)$ & $0,44(0,39-0,49)$ & $0,742^{* *}$ \\
\hline \multicolumn{4}{|c|}{ Sensibilidad a las pruebas intraepidérmicas, n (\%) } \\
\hline Sí & $21(80,8)$ & $0,45(0,42-0,54)$ & \\
\hline No & $5(19,2)$ & $0,33(0,28-0,56)$ & $0,111^{* *}$ \\
\hline \multicolumn{4}{|c|}{ Gravedad de las exacerbaciones de asma (GINA), n (\%) } \\
\hline Leve-moderada & $22(84,6)$ & $0,44(0,39-0,46)$ & \\
\hline Grave & $4(15,4)$ & $0,57(0,49-0,69)$ & $0,009^{* *}$ \\
\hline
\end{tabular}

IRA: Infección de las vías respiratorias altas. PCR: Proteína C reactiva. GINA: Iniciativa global para el asma.

${ }^{*}$ Mediana (intervalo intercuartílico). ${ }^{* *}$ Prueba U de Mann Whitney 
de oxígeno y la concentración de IMA $(p=0,063)$. Se observó una correlación positiva entre la concentración de IMA y la gravedad de las exacerbaciones de asma $(\mathrm{r}=0,498 ; p=0,010)$ (Figura 1).

\section{DISCUSIÓN}

Encontramos que las concentraciones de IMA en los niños con exacerbación del asma eran más elevadas que en los controles. Es posible que haya hipoxia durante la exacerbación, en función de su gravedad. La mayoría de las veces, se observa hipoxia en las exacerbaciones graves, y la saturación puede disminuir por debajo del 90\%. ${ }^{1}$ Esperábamos una concentración de IMA más alta durante la exacerbación del asma, ya que la IMA está relacionada con la isquemia y, coincidentemente con esta hipótesis, encontramos en nuestro estudio que las concentraciones de IMA eran más elevadas en los niños con asma exacerbada que en los controles. En la bibliografía en inglés, no hay estudios que evalúen las concentraciones de IMA en los pacientes asmáticos. En nuestro país, Turquía,
Karagol et al. ${ }^{9}$ llevaron a cabo un estudio en el que evaluaron las concentraciones de IMA en niños asmáticos durante la exacerbación del asma y 14 días después. Al igual que en el estudio de Karagol, encontramos que las concentraciones de IMA en los pacientes con exacerbación del asma eran significativamente más elevadas que en los controles. Y aunque las concentraciones de IMA fueron más bajas durante el período de estabilidad que durante las exacerbaciones, la diferencia no fue estadísticamente significativa en nuestro estudio. Las concentraciones de IMA en los pacientes asmáticos asintomáticos fueron notablemente más elevadas que en los controles. Karagol et al. ${ }^{9}$ mostraron concentraciones de IMA más bajas, aunque no estadísticamente significativas, en los pacientes asmáticos asintomáticos que en los controles $(p=0,076)$. Esta discrepancia con nuestro estudio quizá se deba a diferencias en el diseño de los estudios. En nuestro estudio, las concentraciones de IMA volvieron a medirse al menos 4 semanas después de las exacerbaciones de asma, mientras que Karagol et al. ${ }^{9}$ repitieron las mediciones

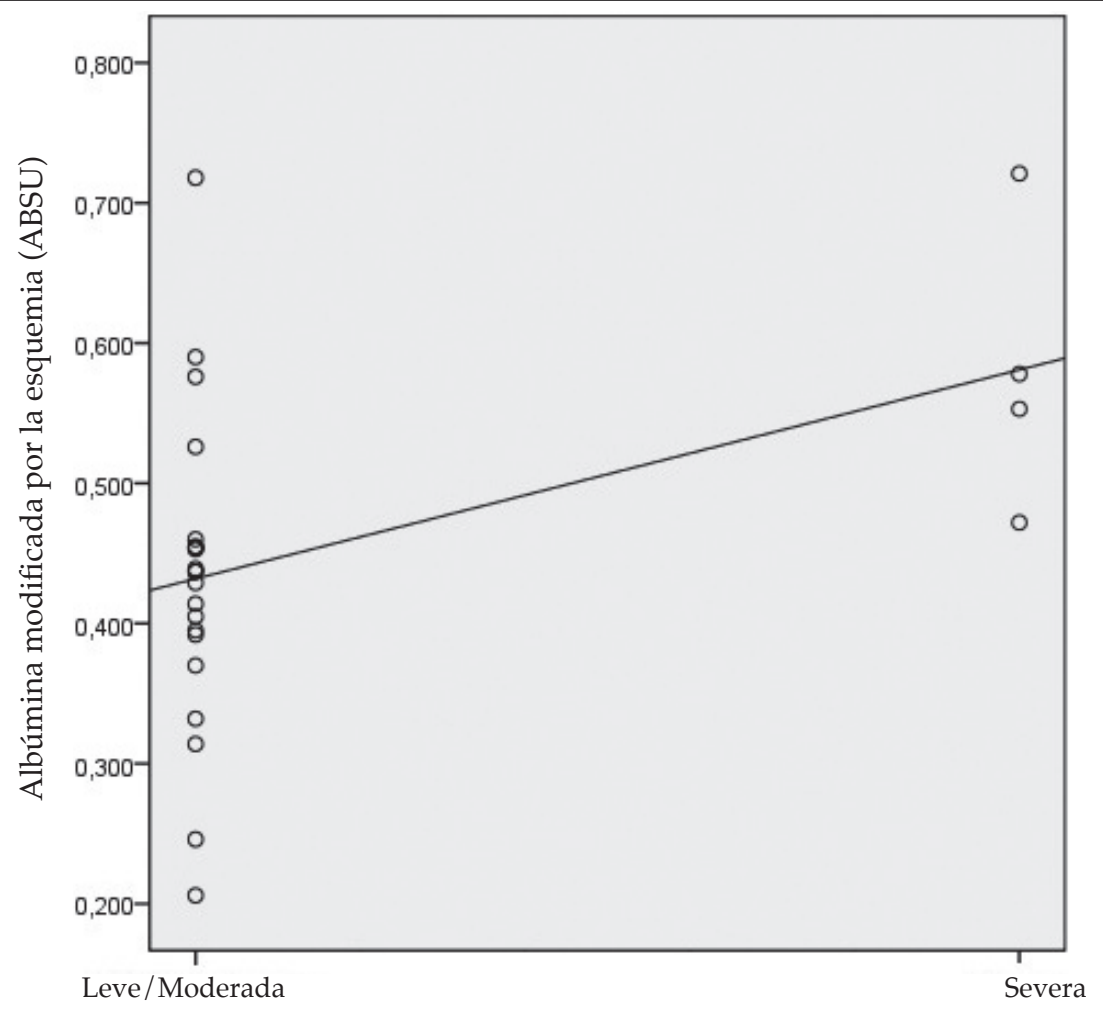

Gravedad de las exacerbaciones 
14 semanas después de las exacerbaciones de asma. Aunque Karagol et al. ${ }^{9}$ no mencionaron el tratamiento que se administró durante la exacerbación, las concentraciones de IMA pueden reducirse notablemente con el tratamiento (como sucede con los esteroides sistémicos) en el período de exacerbación. Más aún, en el estudio de Karagol et al. ${ }^{9}$ se incluyeron solo pacientes con exacerbaciones leves a moderadas, mientras que, en nuestro estudio, participaron varios pacientes con exacerbaciones graves.

Aunque en un principio se pensó que las concentraciones de IMA eran específicas para la isquemia, se ha demostrado que algunos factores, como la acidosis, los efectos de los radicales superóxido, la rotura de la membrana dependiente de la energía y la exposición a iones libres de hierro y cobre, provocan la formación de IMA. ${ }^{4}$ Además de las enfermedades relacionadas con la isquemia y en función de estos factores, se ha encontrado que las concentraciones de IMA aumentan en los casos de enfermedades inflamatorias. ${ }^{5-8}$ Además de la exacerbación del asma, en los pacientes asmáticos, la inflamación de las vías respiratorias continúa incluso después del período estable. ${ }^{1}$ Se supone que las concentraciones de IMA que, según se demostró, aumentan con las enfermedades inflamatorias, aumentarán también en los pacientes asmáticos durante el período estable. Coincidentemente con esta teoría, observamos que las concentraciones de IMA durante el asma estable fueron más altas que en los controles. Can et al., ${ }^{6}$ evaluaron las concentraciones de IMA en pacientes con EPOC, que es otro tipo de enfermedad inflamatoria crónica de las vías respiratorias, en un estudio en el que participaron 51 pacientes con EPOC y 45 controles sanos, y encontraron que las concentraciones de IMA eran más elevadas en los pacientes con EPOC que en el grupo de referencia. Estos resultados sugieren que la IMA puede utilizarse para evaluar la inflamación además de la hipoxia en enfermedades inflamatorias de las vías respiratorias como el asma y la EPOC. Nuestro estudio demostró que las concentraciones de IMA en los niños asmáticos que recibieron tratamiento antiinflamatorio eran más bajas (aunque no de manera estadísticamente significativa) que la de los niños que no habían recibido tratamiento antiinflamatorio, lo que confirma nuestra hipótesis. Nuevos estudios nos permitirán tomar decisiones más acertadas sobre este tema.

Evaluamos la gravedad de las exacerbaciones mediante las clasificaciones GINA y MPIS.
Observamos una correlación fronteriza positiva entre los valores del MPIS y las concentraciones de IMA. De manera similar, observamos una correlación positiva entre la clasificación GINA y las concentraciones de IMA, aunque contábamos con un número limitado de pacientes con exacerbaciones graves. Ese resultado podría deberse a un mayor grado de hipoxia e inflamación como consecuencia de la gravedad de la exacerbación. Lamentablemente, no pudimos evaluar el estado de la inflamación. Observamos una relación negativa pero no estadísticamente significativa entre la saturación de oxígeno y la concentración de IMA ( $\mathrm{r}=-0,370 ; p=0,063)$. En el estudio de Karagol et al., ${ }^{9}$ no hubo correlación entre la gravedad de la exacerbación y las concentraciones de IMA. De manera similar, tampoco hubo correlación entre la saturación de oxígeno y las concentraciones de IMA. Sin embargo, Karagol et al., ${ }^{9}$ solo evaluaron a niños con exacerbaciones de asma leves y moderadas, y no con exacerbaciones graves. En nuestro estudio, los niños con exacerbaciones leves y moderadas se incluyeron en un mismo grupo.

Nuestro estudio presenta varias limitaciones. En primer lugar, el número de pacientes fue limitado, puesto que nuestro estudio se desarrolló en un solo centro y fue transversal, con instalaciones limitadas. El tamaño calculado de la muestra no se alcanzó debido a dificultades económicas.

Es más, no pudimos evaluar la función pulmonar de los pacientes. En tercer lugar, no pudimos evaluar la inflamación de las vías respiratorias mediante la medición del óxido nítrico (NO) exhalado, ni evaluar la citología del esputo, etc. Pese a todas estas limitaciones, creemos que nuestro estudio es importante, puesto que incluye datos valiosos que disipan la deficiencia de la bibliografía.

\section{CONCLUSIÓN}

Encontramos que las concentraciones de IMA en los niños con exacerbación del asma eran más elevadas que en los controles. Además, en comparación con los controles, las concentraciones de IMA resultaron más altas en el período de asma estable. Se demostraron correlaciones positivas entre las concentraciones de IMA y la gravedad de la exacerbación del asma. La IMA puede ser útil para evaluar tanto la inflamación como la gravedad de la exacerbación en los niños asmáticos. Sin embargo, es necesario llevar a cabo estudios más integrales sobre este tema. 


\section{REFERENCIAS}

1. Global Initiative for Asthma: Global Strategy for Asthma Management and Prevention. Actualización: agosto de 2015. [Último acceso: 17 de noviembre de 2016]. Disponible en:http: / / ginasthma.org/wp-content/uploads/2016/01/ GINA_Report_2015_Aug11-1.pdf

2. Bar-Or D, Lau E, Winkler JV. A novel assay for cobaltalbumin binding and its potential as a marker for myocardial ischemia-a preliminary report. J Emerg Med 2000; 19(4):311-5.

3. Toker A, Aribas A, Yerlikaya FH, et al. Serum and saliva levels of ischemia-modified albumin in patients with acute myocardial infarction. J Clin Lab Anal 2013; 27(2):99-104.

4. Sbarouni E, Georgiadou P, Voudris V. Ischemia modified albumin changes - review and clinical implications. Clin Chem Lab Med 2011; 49(2):177-84.

5. Toker A, Karatas Z, Altın H, et al. Evaluation of serum ischemia modified albumin levels in acute rheumatic fever before and after therapy. Indian J Pediatr 2014; 81(2):120-5.
6. Can U, Yerlikaya FH, Yosunkaya S. Role of oxidative stress and serum lipid levels in stable chronic obstructive pulmonary disease. J Chin Med Assoc 2015; 78(12):702-8.

7. Kaplan M, YukselM, AtesI, etal. Isischemia modified albumin a disease activity marker for inflammatory bowel diseases? J Gastroenterol Hepatol 2016; 31(6):1120-5.

8. Ozyazgan S, Andican G, Erman H, et al. Relation of protein oxidation parameters and disease activity in patients with Behçet's disease. Clin Lab 2013; 59(7-8):819-25.

9. Karagol C, Ertoy Karagol HI, Kiyici A, et al. Ischemia Modified Albumin Levels In Children With Acute Asthma Attack. Turkish J Pediatr Dis 2012; 6(3):161-6.

10. Mehmetoglu I, Kurban S, Yerlikaya FH, et al. Obesity is an independent determinant of ischemia-modified albumin. Obes Facts 2012; 5(5):700-9.

11. Carroll CL, Sekaran AK, Lerer TJ, et al. A modified pulmonary index score with predictive value for pediatric asthma exacerbations. Ann Allergy Asthma Immunol 2005; 94(3):355-9. 